\title{
The Application of UG Secondary Development to the Calculation of Dimension Chain
}

\author{
Ji Li ${ }^{1, a}$, Liu Yongping ${ }^{2, b}$, Li Yan ${ }^{3, c}$, Feng Yuewei ${ }^{4, b}$ \\ ${ }^{1,2}$ College of electromechanical engineering, Shenyang Aerospace University Shenyang, China \\ ${ }^{3}$ Qindao College, Qingdao Technological University ,Qingdao ,China \\ ${ }^{4}$ AVIC Shenyang Liming Aero-engine(group) Corporation LTD. Shenyang, China \\ ajili_rory@126.com , ${ }^{b} 710622852 @ q q . c o m,{ }^{c}$ ly_happyhappy@126.com, dfyw0600685@qq.com
}

Keywords: dimension chain, UG, secondary development, VB.NET

\begin{abstract}
In order to reduce the complex processes and errors when we calculate the dimension chain by manual, a reasonable method of calculating dimension chain is employed and some computer aided analysis models are established, which based on the mathematical models of dimension chain calculation. In the UG platform, we developed a completed dimension chain calculation analysis software with VB.NET secondary development, and applied to dimension chain check, and significantly saving the time spent in the process of calculating the dimension chain by the staff.
\end{abstract}

\section{Introduction}

The dimension chain is the effective tool of analyzing and calculating dimension process, and plays an important role in the machining process and ensuring equipment precision. In the process of machining and current analyzing, we often encounter complex problem of analyzing and calculating dimension chain. In the traditional process of the analyzing and calculating dimension chain, designers generally solved the question through manually calculating the equation of dimension chain. Often, the more loops of dimension chain, the worse character increasing or decreasing, leading to the mistake of calculating results of the unknown loop. And the whole process must be done lots of work, cost more time.

In order to solve the above problems, improve product digital level of management, by using a kind of effective method of the computer aided process design. So we propose a calculation software of UG NX secondary development to solve the problem of calculation of dimension chain. It not only has fundamentally solved the problem of the efficiency of process design , the long working cycle and the high cost, but also has made the process design personal liberated from complicated job, and put attention to improving the quality of the products and technology level, and improve creativeness of the people of designing process.

\section{The method of calculating dimension chain}

The extreme value method of calculating dimension chain

This method expresses the relations of among the composing loop and the closed loop when the composing loop appears extreme value. This calculation method is used when each the component loop appears extreme at the same time, the possibility is too little, so the more conservative, the method of calculation can ensure the quality, and special simple be used, so it widely applied in the production, especially in the aerospace industry factory .

The basic formula of extreme value method:

$$
A_{0}=\sum_{i=1}^{m} \vec{A}_{i}-\sum_{j=m+1}^{n-1} \bar{A}_{j}
$$




$$
\begin{aligned}
& E_{S} A_{0}=\sum_{i=1}^{m} E_{S} \vec{A}_{i}-\sum_{j=m+1}^{n-1} E_{i} \bar{A}_{j} \\
& E_{i} A_{0}=\sum_{i=1}^{m} E_{i} \vec{A}_{i}-\sum_{j=m+1}^{n-1} E_{s} \bar{A}_{j}
\end{aligned}
$$

Among them: $A_{0}, \bar{A}_{i}$ and $\bar{A}_{j}$ are the basic of the closed loop, the increasing loop and the decreasing loop; $E_{S} A_{0}, E_{S} \bar{A}_{i}$ and $E_{i} \bar{A}_{j}$ are the upper deviation of the closed loop, the increasing loop and the decreasing loop ; $E_{i} A_{0}, E_{i} \vec{A}_{i}$ and $E_{s} \bar{A}_{j}$ are the lower deviation of the closed loop, the increasing loop and the decreasing loop; $\mathrm{m}$ is the number of the increasing loop of dimension chain; $\mathrm{n}$ is the total number of dimension chain loops[5];

When the number of loop of the dimension chain is less, it can be directly judged character of increasing or decreasing by the definition of increase and decrease. For several more dimension chain loops, in order to quickly and correctly judge the increasing or decreasing loop through planting a one-way arrows of any direction from the closed loop, and then in sequence to draw all of the composing loop arrows from the end direction of connecting arrow. The contrary direction loop compared to the closed loop arrow is increasing loop, and the same direction compared to the closed loop arrow is decreasing loop. As shown in the Fig.1,it is a simple dimension chain, A4 is the closed loop,A1,A2andA3 is the composing loop of this closed loop. And A1 is the increasing loop, A2andA3 are decreasing loops. As a result: A4=A1-A2-A3, the upper and lower deviation could be calculated by the extreme value method[1].

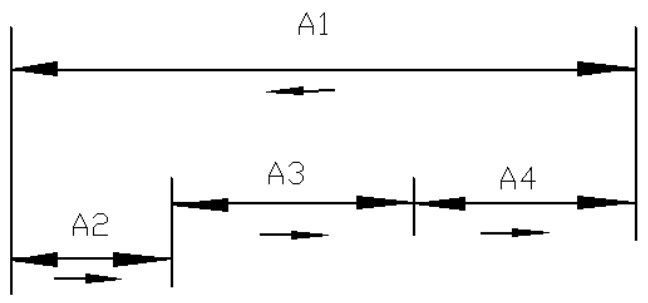

Fig .1 Process dimension chain

\section{The calculation of UG secondary development process dimension chain}

In the application of the computer aided calculating dimension chain, we must correctly confirm the right relations of loops, according to machining process of the part or machine assembling process and the relationship of dimension chain confirmed the closing link, composing loop, to ensure the validity of the calculation of the dimension chain. In order to draw the correct dimension chain figure, for the first intuitional 3D parts, we carry through the intuitive software UG NX function two-dimensional projection, The dimension of each plane within the specified coordinates projection, the dimension information of 3D dimension chain equivalent exchange into the information of two-dimensional dimension chain, and in the two-dimensional plane, confirm the closed loop, composing loop, and confirm increasing or decreasing of the composing loop. Fig. 2 is flow chart of the solution of the dimension chain[2]. 


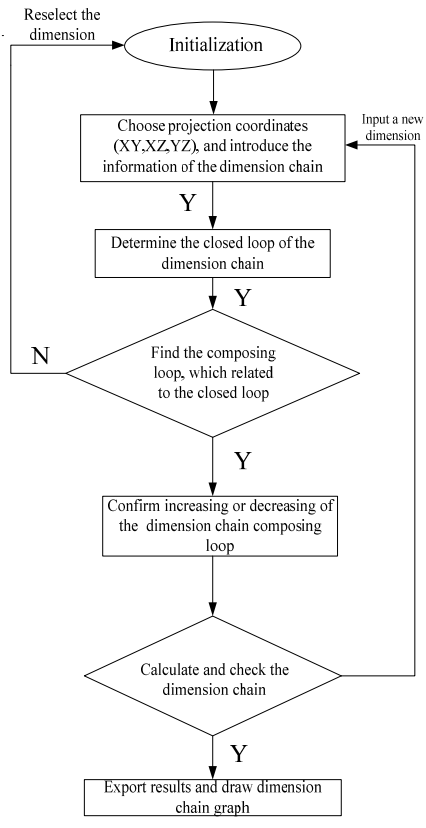

Fig.2 Flow chart

\section{Software development and application}

VB.NET (Visual Basic.NET)is evolved from Visual Basic language, and designed to a kind of application language in other to efficient to generate type safety and object-oriented, inherited advantages of the Visual Basic programming which have safety and language interoperability aspect. Siemens PLM Software Company provided the digital product development system, and supported NX VB.NET programming language. For automation and user-defined product, Siemens development system provided a broad and flexible environment, made simple tool and advanced programming toolkit[3].

According to the simplicity of vb.net software development, we choose vb.net UG NX to a secondary development, because the content are many, here we briefly introduce the main content of development and the process of calculating dimension chain. As shown in Fig.4, it is the main interface of calculating the closed loop of dimension chain. First we must confirm the closed loop which is indirectly obtained and calculated in the machining process, second find out the entire composing loop that related to the closed loop, last confirm increasing or decreasing character of the composing loop, so we can calculate the closed loop of dimension chain. The interface is divided into four parts, The above section mainly displays information of the closed loop, including the basic sizes, upper and lower deviation of closed loops.

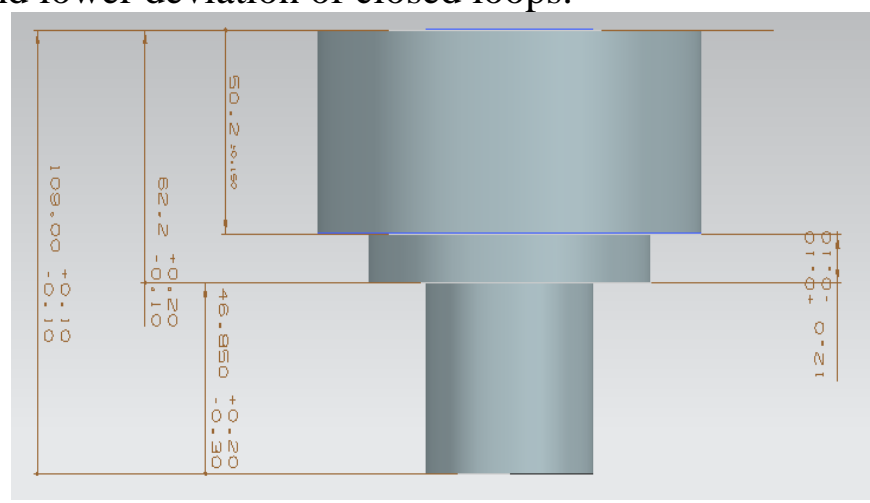

Fig.3 The dimension chain figure of 3D part

Intermediate part displays information of the composing loop, the dimension information of composing loop shows in the list box, and can choose the increasing or decreasing character of loop through following box. In the below interface there are four buttons that are needed user's 
operations. The right part is the view conversion buttons, it is mainly changed the view of UG 3D part. If we would calculate dimension chain in one view, we must to be changed view direction to find conveniently the information of the composing loop. As shown in Fig.3, it is a part two-dimension plane figure which expresses information of 3D dimensional chain equivalent exchange into information of the dimension chain[4].

Click on the[selected the component loop] button, Choose the composing loop of dimension chain ,importing dimensional chain data , confirming the increasing or decreasing of the composing loop, and click on [calculated the closed loop] button to calculate basic sizes ,upper and lower deviation of the closed loop, And judging the calculation result. As shown in Fig.4:

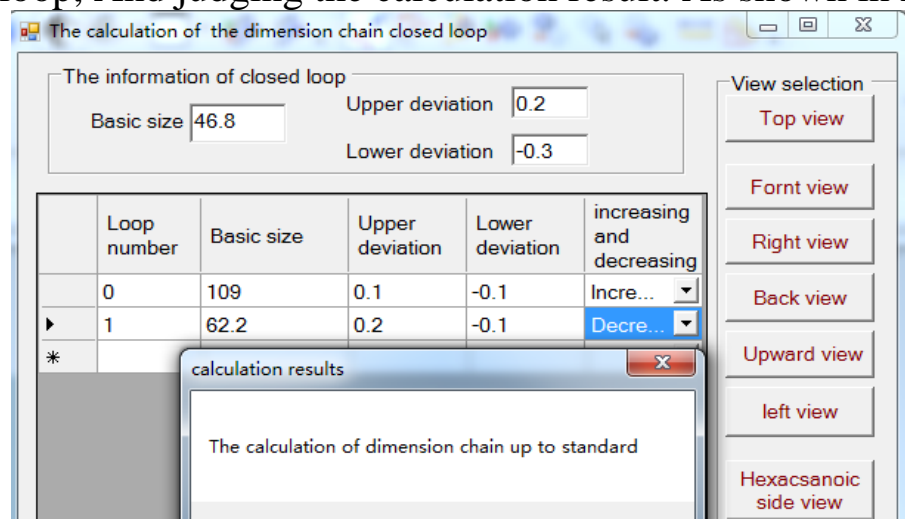

Fig.4 The result and judgment of calculation

The calculation results of the dimension chain have been shown in the window, and we could judge the computational results. it provided a reference for process designer, with saving time of calculating dimension chain by manual for process designer. Not only increasing efficiency of technical procedure layout of process design personal, also ensuring the accuracy of the calculation of the process dimension chain.

\section{Conclusion}

This paper is that UG NX secondary development of dimension chain computing applied in engineering practice, by using the VB.NET, successfully achieving the process of computer-aided calculating dimension chain, and completing the process of the automatic calculating the basic size, upper and lower of deviation. The calculated results are accurate and reliable and fast, good performance of man-machine interaction. It greatly saves time of process designer calculating dimension chain by manual, greatly enhances work efficiency of the process designer, shorten the time of preparation, thus speeding up production of new products.

\section{References}

[1] Zhang J Z, Zhu Y, Yu C. Mechanical manufacture technology [M]. Publishing house of defense industry, 2008.

[2] Cheng C H. Calculation software system development and research of technology dimension chain [D]. Tong Ji University, 2004.

[3] Wei X L, Wang J S. Calculation software system development of line dimension chain based on visual basic [J].Engineering \& Automation, 2005, (2).

[4] Wang X M, Liu S Q. The program design method of dimension chain calculation based on VB

[J]. Ceramic Research and Vocational Education, 2008,(1).

[5] Wang J S, Zhao Z Y. Computing calculation and analysis of dimension chain [J]. Manufacturing Technology \& Machine Tool, 1994,(5). 